\title{
A new subclass of analytic functions
}

\author{
Hesam Mahzoon $^{1}$ (D), Janusz Sokół ${ }^{* 2}$ (DD \\ ${ }^{1}$ Department of Mathematics, Islamic Azad University, West Tehran Branch, Tehran, Iran \\ ${ }^{2}$ University of Rzeszów, College of Natural Sciences, ul. Prof. Pigonia 1, 35-310 Rzeszów, Poland
}

\begin{abstract}
In the present paper, we introduce a class $\mathcal{B}_{\theta}(\alpha, \beta)$ of functions, analytic in $|z|<1$, such that $f(0)=0, f^{\prime}(0)=1$ and

$$
\alpha<\operatorname{Re}\left(f^{\prime}(z)+\frac{1+e^{i \theta}}{2} z f^{\prime \prime}(z)\right)<\beta \quad(|z|<1),
$$

where $\theta \in(-\pi, \pi], 0 \leq \alpha<1$ and $\beta>1$. Integral representation, differential subordination results and coefficient estimates are considered. Also Fekete-Szegö coefficient functional associated with the $k$-th root transform $\left[f\left(z^{k}\right)\right]^{1 / k}$ for functions in the class $\mathcal{B}_{\theta}(\alpha, \beta)$ is investigated.
\end{abstract}

Mathematics Subject Classification (2010). 30C45, 30C50

Keywords. analytic functions, univalent functions, Carathéodory functions, differential subordination, Fekete-Szegö inequality

\section{Introduction}

Let $\mathcal{A}$ be the class of functions of the form

$$
f(z)=z+\sum_{n=2}^{\infty} a_{n} z^{n}
$$

in the open unit disk $\Delta=\{z \in \mathbb{C}:|z|<1\}$. The most familiar subclass of $\mathcal{A}$ consists of univalent functions and it is denoted by $\mathcal{S}$. For a univalent function $f$ of the form (1.1), the $k$-th root transform is defined by

$$
F(z)=\left[f\left(z^{k}\right)\right]^{1 / k}=z+\sum_{n=1}^{\infty} b_{k n+1} z^{k n+1} \quad(z \in \Delta) .
$$

Let $\mathcal{P}$ denote the class of functions $q$ of the form

$$
q(z)=1+\sum_{n=1}^{\infty} q_{n} z^{n}, \quad(z \in \Delta)
$$

\footnotetext{
*Corresponding Author.

Email addresses: mahzoon_hesam@yahoo.com (H. Mahzoon), jsokol@ur.edu.pl (J. Sokół)

Received: 04.04.2019; Accepted: 16.09.2019
} 
such that $\operatorname{Re}\{q(z)\}>0$, for $z \in \Delta$. Functions in $\mathcal{P}$ are sometimes called Carathéodory functions. We also denote by $\mathcal{B}$ the class of analytic functions of the form

$$
w(z)=\sum_{n=0}^{\infty} c_{n} z^{n}, \quad(z \in \Delta)
$$

such that $|w(z)|<1$ for $z \in \Delta$. If $f$ and $g$ are two functions in $\mathcal{A}$, we say that $f$ is subordinate to $g$, written $f(z) \prec g(z)$, if there exists a $w \in \mathcal{B}$ such that $f(z)=g(w(z))$ for all $z \in \Delta$. Furthermore, if the function $g$ belongs to $\mathcal{S}$, then we have the following equivalence:

$$
f(z) \prec g(z) \Leftrightarrow(f(0)=g(0) \text { and } \quad f(\Delta) \subset g(\Delta)) .
$$

For functions $f$ given by (1.1) and $g \in \mathcal{A}$ given by

$$
g(z)=z+\sum_{n=2}^{\infty} b_{n} z^{n} \quad(z \in \Delta),
$$

their Hadamard product (or convolution), denoted by $f * g$, is defined by

$$
(f * g)(z):=z+\sum_{n=2}^{\infty} a_{n} b_{n} z^{n}=:(g * f)(z) \quad(z \in \Delta) .
$$

We now recall that the function class $\mathcal{R}$ is given by

$$
\mathcal{R}=\left\{f \in \mathcal{A}: \quad \operatorname{Re}\left(f^{\prime}(z)+z f^{\prime \prime}(z)\right)>0, \quad z \in \Delta\right\} .
$$

This class was investigated by Chichra [2] and also by Singh and Singh [14]. Another function class $\mathcal{R}_{\alpha}, 0 \leq \alpha<1$, given by

$$
\mathcal{R}_{\alpha}=\left\{f \in \mathcal{A}: \quad \operatorname{Re}\left(f^{\prime}(z)+z f^{\prime \prime}(z)\right)>\alpha, \quad z \in \Delta\right\},
$$

was considered by Silverman [12]. Recently, Silverman and Silvia [13] considered the following classes of functions:

$$
\mathfrak{L}_{\theta}:=\left\{f \in \mathcal{A}: \quad \operatorname{Re}\left(f^{\prime}(z)+\frac{1+e^{i \theta}}{2} z f^{\prime \prime}(z)\right)>0, \quad z \in \Delta\right\}
$$

and

$$
\mathfrak{L}_{\theta}(b):=\left\{f \in \mathcal{A}: \quad\left|f^{\prime}(z)+\frac{1+e^{i \theta}}{2} z f^{\prime \prime}(z)-b\right|<b, \quad z \in \Delta\right\},
$$

for some and $b>1 / 2$ and $-\pi<\theta \leq \pi$. Clearly, if $b \rightarrow \infty$, then $\mathfrak{L}_{\theta}(b) \rightarrow \mathfrak{L}_{\theta}$.

In [8] Kuroki and Owa introduced the class $\mathcal{S}(\alpha, \beta),(\alpha<1<\beta)$, of all functions $f \in \mathcal{A}$ satisfying the following two-sided inequality

$$
\alpha<\operatorname{Re}\left(\frac{z f^{\prime}(z)}{f(z)}\right)<\beta \quad(z \in \Delta) .
$$

In [4], Kargar et al. introduced the class $\mathcal{V}(\alpha, \beta)$ as follows:

$$
\mathcal{V}(\alpha, \beta):=\left\{f \in \mathcal{A}: \alpha<\operatorname{Re}\left\{\left(\frac{z}{f(z)}\right)^{2} f^{\prime}(z)\right\}<\beta, z \in \Delta\right\},
$$

for some $0 \leq \alpha<1$ and $\beta>1$. Also, they (see [5]) introduced the class $\mathcal{M}(\delta)$ including all functions $f \in \mathcal{A}$ such that

$$
1+\frac{\delta-\pi}{2 \sin \delta}<\operatorname{Re}\left\{\frac{z f^{\prime}(z)}{f(z)}\right\}<1+\frac{\delta}{2 \sin \delta} \quad(z \in \Delta)
$$

where $\pi / 2 \leq \delta<\pi$.

In this work, motivated by the above definitions, we define a new subclass of analytic functions related to functions of positive real part. 
Definition 1.1. Assume that $\theta \in(-\pi, \pi], 0 \leq \alpha<1$ and $\beta>1$. A function $f \in \mathcal{A}$ is said to be in the class $\mathcal{B}_{\theta}(\alpha, \beta)$ if it satisfies the following two-sided inequality

$$
\alpha<\operatorname{Re}\left(f^{\prime}(z)+\frac{1+e^{i \theta}}{2} z f^{\prime \prime}(z)\right)<\beta, \quad(z \in \Delta) .
$$

We put $\mathcal{B}_{0}(\alpha, \beta)=\mathcal{B}(\alpha, \beta)$. We remark that the class $\mathcal{B}_{0}(0, \beta \rightarrow \infty) \equiv \mathcal{R}, \mathcal{B}_{0}(\alpha, \beta \rightarrow$ $\infty) \equiv \mathcal{R}_{\alpha}$. Also $\mathcal{B}_{\theta}(0, \beta \rightarrow \infty) \equiv \mathcal{L}_{\theta}$ where $\theta \in(-\pi, \pi]$.

In order to prove our results, we need the following lemmas.

Lemma $1.2([11])$. Let $q(z)=\sum_{n=1}^{\infty} C_{n} z^{n}$ be analytic and univalent in $\Delta$, and suppose that $q$ maps $\Delta$ onto a convex domain. If $p(z)=\sum_{n=1}^{\infty} A_{n} z^{n}$ is analytic in $\Delta$ and satisfies the following subordination

$$
p(z) \prec q(z) \quad(z \in \Delta),
$$

then

$$
\left|A_{n}\right| \leq\left|C_{1}\right| \quad n \geq 1 .
$$

The next lemma is due to Hallenbeck and Ruscheweyh.

Lemma 1.3 ([3]). Let $h$ be a convex univalent function with $h(0)=a$ and let $\gamma \in \mathbb{C}$ with $\operatorname{Re}\{\gamma\} \geq 0$. If the function $p(z)$ given by $p(z)=a+p_{n} z^{n}+p_{n+1} z^{n+1}+\cdots$ is analytic in $\Delta$ and

$$
p(z)+\frac{1}{\gamma} z p^{\prime}(z) \prec h(z) \quad(z \in \Delta),
$$

then

$$
p(z) \prec q(z) \prec h(z) \quad(z \in \Delta)
$$

where

$$
q(z)=\frac{\gamma}{n z^{\gamma / n}} \int_{0}^{z} h(\xi) \xi^{\gamma / n-1} d \xi .
$$

The result is best possible, in the sense that if $p$ satisfies (1.7) and $p \prec q_{1}$, then $q \prec q_{1}$.

Finally, we need the following Lemma 1.4 due to Keogh and Merkes (see [7]).

Lemma 1.4. Let the function g given by

$$
g(z)=1+c_{1} z+c_{2} z^{2}+\cdots,
$$

be in the class $\mathcal{P}$. Then, for any complex number $\mu$

$$
\left|c_{2}-\mu c_{1}^{2}\right| \leq 2 \max \{1,|2 \mu-1|\} .
$$

The result is sharp for the function g given by

$$
g(z)=\frac{1+z}{1-z} \quad \text { or } \quad g(z)=\frac{1+z^{2}}{1-z^{2}} .
$$

In this paper some properties of the function class $\mathcal{B}_{\theta}(\alpha, \beta)$ including, integral representation, differential subordination results and coefficient estimates are investigated. Furthermore, Fekete-Szegö coefficient functional associated with the $k$-th root transform $\left[f\left(z^{k}\right)\right]^{1 / k}$ is considered. 


\section{Main results}

We begin this section with the following lemma that will be useful. Indeed, it presents a necessary and sufficient condition for functions to be in the class $\mathcal{B}_{\theta}(\alpha, \beta)$.

Lemma 2.1. Assume that $f \in \mathcal{A}, 0 \leq \alpha<1, \beta>1$ and $\theta \in(-\pi, \pi]$. Then $f \in \mathcal{B}_{\theta}(\alpha, \beta)$, if and only if

$$
f^{\prime}(z)+\frac{1+e^{i \theta}}{2} z f^{\prime \prime}(z) \prec P_{\alpha, \beta}(z) \quad(z \in \Delta)
$$

where

$$
P_{\alpha, \beta}(z):=1+\frac{\beta-\alpha}{\pi} i \log \left(\frac{1-e^{2 \pi i \frac{1-\alpha}{\beta-\alpha}} z}{1-z}\right) .
$$

Proof. Note that the function $P_{\alpha, \beta}$ defined by $(2.2)$ is convex univalent function in $\Delta$ and maps $\Delta$ onto a convex domain

$$
\Omega_{\alpha, \beta}=\{w \in \mathbb{C}: \alpha<\operatorname{Re}\{w\}<\beta\},
$$

conformally (see [8]). By (1.6), $\left\{f^{\prime}(z)+\left[\left(1+e^{i \theta}\right) / 2\right] z f^{\prime \prime}(z)\right\}$ lies in the strip $\Omega_{\alpha, \beta}$ and it is known that $P_{\alpha, \beta}(\Delta)=\Omega_{\alpha, \beta}$. Because $P_{\alpha, \beta}$ is univalent then by the subordination principle, we get $(2.1)$.

It is easy to see the function $P_{\alpha, \beta}$ has the form

$$
P_{\alpha, \beta}(z)=1+\sum_{n=1}^{\infty} B_{n} z^{n}
$$

where

$$
B_{n}=\frac{\beta-\alpha}{n \pi} i\left(1-e^{2 n \pi i \frac{1-\alpha}{\beta-\alpha}}\right) \quad(n=1,2, \ldots) .
$$

Lemma 2.2. Let $P_{\alpha, \beta}$ be defined as (2.2). Then the function

$$
\lambda(z)=\int_{0}^{1} P_{\alpha, \beta}\left(z t^{1 / \gamma}\right) d t \quad(\operatorname{Re}\{\gamma\}>0, z \in \Delta),
$$

is convex in $\Delta$.

Proof. Define

$$
\psi_{\gamma}(z):=\int_{0}^{1} \frac{1}{1-z t^{1 / \gamma}} d t=\sum_{n=0}^{\infty} \frac{\gamma}{n+\gamma} z^{n} .
$$

The function $\psi_{\gamma}(z)$ is convex in $\Delta$ when $\operatorname{Re}(\gamma)>0$ (see [9]). From (2.7) we obtain

$$
\begin{aligned}
P_{\alpha, \beta}(z) * \psi_{\gamma}(z) & =P_{\alpha, \beta}(z) * \int_{0}^{1} \frac{1}{1-z t^{1 / \gamma}} d t \\
& =\int_{0}^{1} P_{\alpha, \beta}\left(z t^{1 / \gamma}\right) d t=: \lambda(z) .
\end{aligned}
$$

On the other hand, since $P_{\alpha, \beta}$ and $\psi_{\gamma}$ are convex univalent functions, by the PòlyaSchoenberg conjecture (this conjecture states that the class of convex univalent functions is preserved under the convolution) that is proved by Ruscheweyh and Sheil-Small (see [10]), the function $\lambda(z)$ is convex univalent in the open unit disk $\Delta$. This completes the proof.

In the sequel, applying the Lemma 2.1, we obtain an integral representation for functions which belong to the class $\mathcal{B}(\alpha, \beta)$. 
Theorem 2.3. Let $\theta \in(-\pi, \pi), 0 \leq \alpha<1$ and $\beta>1$. Then $f \in \mathcal{B}_{\theta}(\alpha, \beta)$ if, and only if there exists $w \in \mathcal{B}$ such that

$$
f(z)=\int_{0}^{z} \frac{\gamma}{\eta^{\gamma}}\left(\int_{0}^{\eta} \xi^{\gamma-1} P_{\alpha, \beta}(w(\xi)) d \xi\right) d \eta \quad\left(z \in \Delta, \gamma=2 /\left(1+e^{i \theta}\right)\right),
$$

where $P_{\alpha, \beta}(z)$ defined by $(2.2)$.

Proof. Let $f \in \mathcal{B}_{\theta}(\alpha, \beta)$. By definition of subordination and by the Lemma 2.1, if $f \in$ $\mathcal{B}_{\theta}(\alpha, \beta)$, then there exists a function $w \in \mathcal{B}$ such that

$$
f^{\prime}(z)+\frac{1+e^{i \theta}}{2} z f^{\prime \prime}(z)=P_{\alpha, \beta}(w(z)) \quad(z \in \Delta) .
$$

From the following equality

$$
f^{\prime}(z)+\frac{1+e^{i \theta}}{2} z f^{\prime \prime}(z)=\frac{1-e^{i \theta}}{2} f^{\prime}(z)+\frac{1+e^{i \theta}}{2}\left(z f^{\prime}(z)\right)^{\prime} \quad(z \in \Delta),
$$

we get that $(2.9)$ is equivalent to

$$
\left(\frac{1-e^{i \theta}}{1+e^{i \theta}}\right) f^{\prime}(z)+\left(z f^{\prime}(z)\right)^{\prime}=\frac{2}{1+e^{i \theta}} P_{\alpha, \beta}(w(z)) \quad(z \in \Delta) .
$$

Assuming

$$
\gamma:=\frac{2}{1+e^{i \theta}} \quad(-\pi<\theta<\pi)
$$

the above relation (2.11) implies that

$$
(\gamma-1) z^{\gamma-1} f^{\prime}(z)+z^{\gamma-1}\left(z f^{\prime}(z)\right)^{\prime}=\gamma z^{\gamma-1} P_{\alpha, \beta}(w(z)) .
$$

Therefore, we find that

$$
\left[z^{\gamma-1}\left(z f^{\prime}(z)\right)\right]^{\prime}=\gamma z^{\gamma-1} P_{\alpha, \beta}(w(z))
$$

which readily yields

$$
z^{\gamma} f^{\prime}(z)=\gamma \int_{0}^{z} \xi^{\gamma-1} P_{\alpha, \beta}(w(\xi)) d \xi .
$$

Integrating once more the equality (2.13), we get (2.8) and concluding the proof.

Let $t \in[0,1]$ and $\phi \in[0,2 \pi)$. By using Theorem 2.3, the function

$$
f(z, \phi, t)=\int_{0}^{z} \frac{\gamma}{\eta^{\gamma}}\left(\int_{0}^{\eta} \xi^{\gamma-1} P_{\alpha, \beta}\left(\frac{e^{i \phi} \xi(\xi+t)}{1+\xi t}\right) d \xi\right) d t \quad(z \in \Delta),
$$

belongs to the class $\mathcal{B}_{\theta}(\alpha, \beta)$.

Theorem 2.4. Let $\theta \in(-\pi, \pi), 0 \leq \alpha<1$ and $\beta>1$. If $f \in \mathcal{B}_{\theta}(\alpha, \beta)$, then

$$
f^{\prime}(z) \prec \int_{0}^{1} P_{\alpha, \beta}\left(z t^{1 / \gamma}\right) d t \prec P_{\alpha, \beta}(z) \quad\left(z \in \Delta, \gamma=2 /\left(1+e^{i \theta}\right)\right),
$$

where $P_{\alpha, \beta}$ defined by (2.2). The result is sharp.

Proof. Since $f \in \mathcal{B}_{\theta}(\alpha, \beta)$, from Lemma 2.1 it follows that (2.1) holds true. If we take $p(z)=f^{\prime}(z)$, then

$$
p(z)+\frac{1}{\gamma} z p^{\prime}(z)=f^{\prime}(z)+\frac{1+e^{i \theta}}{2} z f^{\prime \prime}(z) \prec P_{\alpha, \beta}(z) \quad(z \in \Delta),
$$

where $\gamma$ is as defined in (2.12). Therefore, applying the Lemma 1.3 and since $P_{\alpha, \beta}$ is a convex univalent function in the open unit disk $\Delta$, we get

$$
p(z) \prec \frac{\gamma}{z^{\gamma}} \int_{0}^{z} \xi^{\gamma-1} P_{\alpha, \beta}(\xi) d \xi \prec P_{\alpha, \beta}(z) \quad(z \in \Delta) .
$$


Now by letting $\xi=z t^{1 / \gamma}$ in the above integral (2.16) the differential chain (2.16) implies that (2.15) holds true for all $z \in \Delta$.

Theorem 2.5. Let $\theta \in(-\pi, \pi), 0 \leq \alpha<1$ and $\beta>1$. If $f \in \mathcal{B}_{\theta}(\alpha, \beta)$, then

$$
\frac{f(z)}{z} \prec \int_{0}^{1} \int_{0}^{1} P_{\alpha, \beta}\left(z r t^{1 / \gamma}\right) d r d t \quad(z \in \Delta)
$$

where $P_{\alpha, \beta}$ defined by (2.2).

Proof. Let that $p(z)=f(z) / z$. Then by using of (2.15) and applying Lemma 2.2, and with a simple computation we have

$$
p(z)+z p^{\prime}(z)=f^{\prime}(z) \prec \int_{0}^{1} P_{\alpha, \beta}\left(z t^{1 / \gamma}\right) d t \quad(z \in \Delta) .
$$

If we apply Lemma 1.3 once more with $\gamma=1$, we conclude that

$$
p(z) \prec \frac{1}{z} \int_{0}^{z} \lambda(\xi) d \xi \prec \lambda(z) \quad(z \in \Delta),
$$

where $\lambda$ defined in (2.6). Now, it is sufficient that we put $\xi=r z$ in the integral in (2.18). In this case, if we take into account (2.6), then the first differential subordination in (2.18) implies that (2.17) holds true and concluding the proof.

Remark 2.6. Since the result of Lemma 1.3 is best possible, it follows that the differential subordinations in (2.15) and (2.17) are also best possible.

\section{On coefficients}

First, we start this section by estimating the coefficients of members of the family $\mathcal{B}_{\theta}(\alpha, \beta)$.

Theorem 3.1. Let $\theta \in(-\pi, \pi], 0 \leq \alpha<1$ and $\beta>1$. Also, let $f(z)=z+\sum_{n=2}^{\infty} a_{n} z^{n}$ belongs to the class $\mathcal{B}_{\theta}(\alpha, \beta)$. Then

$$
\left|a_{n}\right| \leq \frac{2 \sqrt{2}(\beta-\alpha)}{n \pi \sqrt{n^{2}+1+\left(n^{2}-1\right) \cos \theta}} \sin \frac{\pi(1-\alpha)}{\beta-\alpha} \quad(n \geq 2) .
$$

Proof. Let $f$ be of the form (1.1) belongs to the class $\mathcal{B}_{\theta}(\alpha, \beta)$. Then by Lemma 2.1, we have

or equivalently

$$
f^{\prime}(z)+\frac{1+e^{i \theta}}{2} z f^{\prime \prime}(z) \prec P_{\alpha, \beta}(z) \quad(z \in \Delta)
$$

$$
1+\sum_{n=1}^{\infty} p_{n} z^{n} \prec 1+\sum_{n=1}^{\infty} B_{n} z^{n} .
$$

From Lemma 1.2, the last differential subordination implies that

$$
\left|p_{n}\right| \leq\left|B_{1}\right| \quad n \geq 1 .
$$

On the other hand, by equating the coefficients of $z^{n}$ on both sides of (3.2), the following relation between the coefficients holds true:

$$
\frac{n}{2}\left[2+(n-1)\left(1+e^{i \theta}\right)\right] a_{n}=p_{n-1} .
$$

Thus, from (3.3), we obtain

$$
\left|a_{n}\right| \leq\left|\frac{B_{1}}{\frac{n}{2}\left[2+(n-1)\left(1+e^{i \theta}\right)\right]}\right|=\frac{\sqrt{2}\left|B_{1}\right|}{n \sqrt{n^{2}+1+\left(n^{2}-1\right) \cos \theta}},
$$

where

This completes the proof.

$$
\left|B_{1}\right|=\frac{2(\beta-\alpha)}{\pi} \sin \frac{\pi(1-\alpha)}{\beta-\alpha} .
$$


With suitable choices of $\alpha, \beta$ and $\theta$, we get the following interesting results.

Corollary 3.2. If $f \in \mathcal{A}$ of the form (1.1) satisfying the following two-sided inequality

$$
\frac{1}{2}<\operatorname{Re}\left(f^{\prime}(z)+z f^{\prime \prime}(z)\right)<\frac{3}{2} \quad(z \in \Delta),
$$

then

$$
\left|a_{n}\right| \leq \frac{2}{\pi n^{2}} \quad(n \geq 2) .
$$

In particular, $\left|a_{2}\right| \leq 1 / 2 \pi$

Corollary 3.3. If $f \in \mathcal{A}$ of the form (1.1) satisfying the following two-sided inequality

$$
0<\operatorname{Re}\left(f^{\prime}(z)+z f^{\prime \prime}(z)\right)<2 \quad(z \in \Delta),
$$

then

In particular, $\left|a_{2}\right| \leq 1 / \pi$.

$$
\left|a_{n}\right| \leq \frac{4}{\pi n^{2}} \quad(n \geq 2)
$$

Recently, many researchers (see e.g. [1], [6]) have considered the Fekete-Szegö functional associated with the $k$-th root transform for several subclasses of analytic functions. In the next result, we consider this problem for functions in the class $\mathcal{B}_{\theta}(\alpha, \beta)$.

Theorem 3.4. Let $\theta \in(-\pi, \pi], 0 \leq \alpha<1$ and $\beta>1$. Also let $f \in \mathcal{B}_{\theta}(\alpha, \beta)$ and $F$ be the $k$-th root transform of $f$ defined by (1.2). Then, for any complex number $\mu$, the following sharp estimates hold true.

$$
\begin{gathered}
\left|b_{2 k+1}-\mu b_{2 k+1}^{2}\right| \leq \frac{2(\beta-\alpha)}{3 k \pi \sqrt{5+4 \cos \theta}} \sin \frac{\pi(1-\alpha)}{\beta-\alpha} \\
\times \max \left\{1,\left|\frac{1+w}{2}-(2 \mu+k-1) \frac{3\left(2+e^{i \theta}\right)(\beta-\alpha) i}{2 k \pi\left(3+e^{i \theta}\right)^{2}}(1-w)\right|\right\},
\end{gathered}
$$

where

$$
w=\exp \left\{\frac{2 \pi i(1-\alpha)}{\beta-\alpha}\right\} .
$$

Proof. Since $f \in \mathcal{B}_{\theta}(\alpha, \beta)$, from definition of subordination, there exists a function $w \in \mathcal{B}$ such that

$$
f^{\prime}(z)+\frac{1+e^{i \theta}}{2} z f^{\prime \prime}(z)=1+\sum_{n=2}^{\infty} \frac{n}{2}\left[2+(n-1)\left(1+e^{i \theta}\right)\right] a_{n} z^{n}=P_{\alpha, \beta}(w(z)) .
$$

Define

$$
\mathfrak{p}(z)=\frac{1+w(z)}{1-w(z)}=1+p_{1} z+p_{2} z^{2}+\cdots .
$$

Since $w \in \mathcal{B}$, it follows that $\mathfrak{p} \in \mathcal{P}$. From (3.6) and (2.4) we have

$$
P_{\alpha, \beta}(w(z))=1+\frac{1}{2} B_{1} p_{1} z+\left(\frac{1}{4} B_{2} p_{1}^{2}+\frac{1}{2} B_{1}\left(p_{2}-\frac{1}{2} p_{1}^{2}\right)\right) z^{2}+\cdots .
$$

Equating the coefficients of $z$ and $z^{2}$ on both sides of (3.5), we get

$$
\left(3+e^{i \theta}\right) a_{2}=\frac{1}{2} B_{1} p_{1},
$$

and

$$
\left(6+3 e^{i \theta}\right) a_{3}=\left(\frac{1}{4} B_{2} p_{1}^{2}+\frac{1}{2} B_{1}\left(p_{2}-\frac{1}{2} p_{1}^{2}\right)\right) .
$$

On the other hand, a simple computation shows that

$$
F(z)=\left[f\left(z^{1 / k}\right)\right]^{1 / k}=z+\frac{1}{k} a_{2} z^{k+1}+\left(\frac{1}{k} a_{3}-\frac{1}{2} \frac{k-1}{k^{2}} a_{2}^{2}\right) z^{2 k+1}+\cdots .
$$


From equations (1.2) and (3.10), we have

$$
b_{k+1}=\frac{1}{k} a_{2} \quad \text { and } \quad b_{2 k+1}=\frac{1}{k} a_{3}-\frac{1}{2} \frac{k-1}{k^{2}} a_{2}^{2} .
$$

Substituting from (3.8) and (3.9) into (3.11), we obtain

$$
b_{k+1}=\frac{B_{1} p_{1}}{2 k\left(3+e^{i \theta}\right)}
$$

and

$$
b_{2 k+1}=\frac{1}{k\left(6+3 e^{i \theta}\right)}\left(\frac{1}{4} B_{2} p_{1}^{2}+\frac{1}{2} B_{1}\left(p_{2}-\frac{1}{2} p_{1}^{2}\right)\right)-\frac{(k-1) B_{1}^{2} p_{1}^{2}}{8 k^{2}\left(3+e^{i \theta}\right)^{2}},
$$

so that

$$
b_{2 k+1}-\mu b_{k+1}^{2}=\frac{B_{1}}{6 k\left(2+e^{i \theta}\right)}\left[p_{2}-\frac{1}{2}\left(1-\frac{B_{2}}{B_{1}}+(2 \mu+k-1) \frac{\left(6+3 e^{i \theta}\right) B_{1}}{2 k\left(3+e^{i \theta}\right)^{2}}\right) p_{1}^{2}\right] .
$$

From (2.5), we have

$$
B_{1}=\frac{\beta-\alpha}{\pi} i\left(1-e^{2 \pi i \frac{1-\alpha}{\beta-\alpha}}\right)
$$

and

$$
B_{2}=\frac{\beta-\alpha}{2 \pi} i\left(1-e^{4 \pi i \frac{1-\alpha}{\beta-\alpha}}\right) .
$$

Putting (3.13) and (3.14) into (3.12) and letting

$$
\mu=\frac{1}{2}\left(1-\frac{B_{2}}{B_{1}}+(2 \mu+k-1) \frac{\left(6+3 e^{i \theta}\right) B_{1}}{2 k\left(3+e^{i \theta}\right)^{2}}\right),
$$

the inequality (3.4) now follows by an application of Lemma 1.4. It is easy to check that the result is sharp for the $k$-th root transforms of the functions $f(z, \phi, 1)$ and $f(z, \phi, 0)$ defined by (2.14).

The problem of finding sharp upper bounds for the coefficient functional $\left|a_{3}-\mu a_{2}^{2}\right|$ for different subclasses of the normalized analytic function class $\mathcal{A}$ is known as the FeketeSzegö problem. If we take $k=1$ in Theorem 3.4, we have.

Corollary 3.5. Let $\theta \in(-\pi, \pi], 0 \leq \alpha<1$ and $\beta>1$. Suppose also that $f \in \mathcal{B}_{\theta}(\alpha, \beta)$. Then, for any complex number $\mu$,

$$
\begin{gathered}
\left|a_{3}-\mu a_{2}^{2}\right| \leq \frac{2(\beta-\alpha)}{3 \pi \sqrt{5+4 \cos \theta}} \sin \frac{\pi(1-\alpha)}{\beta-\alpha} \\
\times \max \left\{1,\left|\frac{1+\exp \left\{\frac{2 \pi i(1-\alpha)}{\beta-\alpha}\right\}}{2}-\mu \frac{3\left(2+e^{i \theta}\right)(\beta-\alpha) i}{\pi\left(3+e^{i \theta}\right)^{2}}\left(1-\exp \left\{\frac{2 \pi i(1-\alpha)}{\beta-\alpha}\right\}\right)\right|\right\} .
\end{gathered}
$$

The result is sharp.

Corollary 3.6. Let $0 \leq \alpha<1$ and $\beta>1$. Suppose also that $f \in \mathcal{B}(\alpha, \beta)$. Then, for any complex number $\mu$,

$$
\begin{gathered}
\left|a_{3}-\mu a_{2}^{2}\right| \leq \frac{2(\beta-\alpha)}{9 \pi} \sin \frac{\pi(1-\alpha)}{\beta-\alpha} \\
\times \max \left\{1,\left|\frac{1+\exp \left\{\frac{2 \pi i(1-\alpha)}{\beta-\alpha}\right\}}{2}-\mu \frac{9(\beta-\alpha) i}{16 \pi}\left(1-\exp \left\{\frac{2 \pi i(1-\alpha)}{\beta-\alpha}\right\}\right)\right|\right\} .
\end{gathered}
$$

The result is sharp. 
Corollary 3.7. If $f \in \mathcal{A}$ of the form (1.1) satisfying the following two-sided inequality

$$
\frac{1}{2}<\operatorname{Re}\left(f^{\prime}(z)+z f^{\prime \prime}(z)\right)<\frac{3}{2} \quad(z \in \Delta),
$$

then

$$
\left|a_{3}-\mu a_{2}^{2}\right| \leq \frac{2}{9 \pi} \max \left\{1, \frac{9|\mu|}{8 \pi}\right\} \quad(\mu \in \mathbb{C}) .
$$

Corollary 3.8. If $f \in \mathcal{A}$ of the form (1.1) satisfying the following two-sided inequality

$$
0<\operatorname{Re}\left(f^{\prime}(z)+z f^{\prime \prime}(z)\right)<2 \quad(z \in \Delta) \text {, }
$$

then

$$
\left|a_{3}-\mu a_{2}^{2}\right| \leq \frac{4}{9 \pi} \max \left\{1, \frac{9|\mu|}{4 \pi}\right\} \quad(\mu \in \mathbb{C}) .
$$

\section{References}

[1] R.M. Ali, S.K. Lee, V. Ravichandran and S. Supramanian, The Fekete-Szegö coefficient functional for transforms of analytic functions, Bull. Iranian Math. Soc. 35, 119-142, 2009.

[2] P.N. Chichra, New subclasses of the class of close-to-convex functions, Proc. Amer. Math. Soc. 62, 37-43, 1977.

[3] D.J. Hallenbeck and St. Ruscheweyh, Subordination by convex functions, Proc. Amer. Math. Soc. 52, 191-195, 1975.

[4] R. Kargar, A. Ebadian, J. Sokól, On subordination of some analytic functions, Sib. Math. J. 57, 599-605, 2016.

[5] R. Kargar, A. Ebadian and J. Sokół, Radius problems for some subclasses of analytic functions, Complex Anal. Oper. Theory 11, 1639-1649, 2017.

[6] R. Kargar, A. Ebadian and J. Sokół, On Booth lemniscate and starlike functions, Anal. Math. Phys. 9, 143-154, 2019.

[7] F.R. Keogh and E. P. Merkes, A coefficient inequality for certain classes of analytic functions, Proc. Amer. Math. Soc. 20, 8-12, 1969.

[8] K. Kuroki and S. Owa, Notes on New Class for Certain Analytic Functions, RIMS Kokyuroku Kyoto Univ. 1772, 21-25, 2011.

[9] St. Ruscheweyh, New criteria for univalent functions, Proc. Amer. Math. Soc. 49, 109-115, 1975.

[10] St. Ruscheweyh and T. Sheil-Small, Hadamard products of schlicht functions and the Polya-Schoenberg conjecture, Comment. Math. Helv. 48, 119-135, 1973.

[11] W. Rogosinski, On the coefficients of subordinate functions, Proc. London Math. Soc. 48, 48-82, 1943.

[12] H. Silverman, A class of bounded starlike functions, Internat. J. Math. Math. Sci. 17, 249-252, 1994.

[13] H. Silverman and E.M. Silvia, Characterizations for subclasses of univalent functions, Math. Japan. 50, 103-109, 1999.

[14] R. Singh and S. Singh, Convolutions properties of a class of starlike functions, Proc. Amer. Math. Soc. 106, 145-152, 1989. 